Johnson \& Wales University

ScholarsArchive@JWU

Health \& Wellness Department Faculty

Publications and Research

$1-2019$

Reliability of Smartphone Inclinometry to Measure Upper Cervical Range of Motion

Paul Ullucci

Follow this and additional works at: https://scholarsarchive.jwu.edu/health_fac

Part of the Medicine and Health Sciences Commons 


\title{
Reliability of Smartphone Inclinometry to Measure Upper Cervical Range of Motion
}

\author{
Paul A. Ullucci, Frank Tudini, and Matthew F. Moran
}

\begin{abstract}
Context: Assessment of upper cervical range of motion (UCROM) and mobility is commonly performed in the clinical setting for patients suffering from headache, neck pain, and vestibular dysfunction. Reliable and reproducible measurement of this motion is often difficult or too expensive to perform in the clinical setting. Smartphone applications using the device's internal gyroscope offer an easy and inexpensive means of measuring UCROM, but their reliability has not been reported in the literature. Objective: To assess the reliability of an inclinometer application installed on 2 different devices (iPhone 6 [IP] and android [AN]) and to measure UCROM in a healthy population. Design: Two examiners assessed passive UCROM. Each examiner was assigned to a specific smartphone, and a repeated-measures design consisting of 3 trials for each examiner-phone was performed. The order of testing was randomized, and the examiners were blinded to UCROM measures. Setting: Laboratory. Participants: A total of 38 subjects (19 females and 19 males; age: 23.8 [1.2] y) without pain or injury to the neck or spine for at least 3 months. Intervention: Each examiner passively flexed the head fully, rotated the head fully in 1 direction, and then in another. Peak rotation measures were recorded from each smartphone. Three trials were performed for each phone, with a 2-minute break between examiners/phones. Main Outcome Measures: Intraclass correlation coefficient using a 2-way mixed, absolute agreement model was obtained (1) between each examiner-phone and (2) within each examiner-phone for the measurements in each rotation direction. Results: Interphone/examiner reliability comparing average peak and total UCROM for each device was excellent (.87, .81). Intraphone/ examiner reliability, determined across 3 trials, was also excellent $(\mathrm{AN}$ right rot. $=.91$, AN left rot. $=.96$; IP right rot. $=.98$, IP left rot. $=.95$ ). Conclusion: UCROM can be reliably measured using a smartphone inclinometer application.
\end{abstract}

Keywords: upper cervical spine, inclinometer, atlanto-axial joint, atlanto-occipital joint

Measurement of cardinal plane cervical range of motion $(\mathrm{CROM})$ is routinely performed in physical therapy. ${ }^{1}$ Cardinal plane motion such as cervical flexion and extension is important clinically but has not been found to identify specific limitations in upper cervical mobility that could be causing or contributing to the patient's pain and dysfunction. ${ }^{1,2}$ Accurate assessment of upper cervical mobility and upper CROM (UCROM) is critical in the evaluation and treatment of those with pathology that can be influenced by the upper cervical spine, such as headache, migraine, vertigo, concussion, and postconcussion syndrome. ${ }^{1-6}$

The cervical flexion and rotation test was developed specifically to assess mobility in the upper cervical spine. It is a commonly used manual examination procedure and considered a reliable measure of UCROM. ${ }^{2,6-8}$ Blanpied et $\mathrm{al}^{2}$ state that because of its high level of specificity (.91), the cervical flexion and rotation test should be performed to rule in cervicogenic headache.

Findings from research investigating vertigo, concussion, postconcussion syndrome, migraines, and cervicogenic headache all state that the upper cervical spine and atlanto-axial and atlantooccipital joints must be evaluated and mobility deficits addressed in patients suffering from these conditions. ${ }^{1-6}$ Despite these recommendations, a limited number of publications describe the prevalence of UCROM mobility deficits in these varying patient populations. ${ }^{6,9}$ This lack of foundational information can be attributed to the high cost and difficulty of accurately measuring UCROM using the currently available gold-standard techniques,

Ullucci and Moran are with the Department of Physical Therapy \& Human Movement Science, Sacred Heart University, Fairfield, CT. Tudini is with Campbell University. Ullucci (ulluccip@sacredheart.edu) is corresponding author. such as video motion analysis, radiographs, and the CROM device (Performance Attainment Associates, Lindstrom, MN). Identifying an inexpensive and readily available tool to measure UCROM would allow researchers and clinicians to effectively measure UCROM and better define upper cervical mobility deficits as they relate to these varied conditions.

Improvements in and the increased availability of portable and inexpensive technologies currently allow smartphone users to assess 3-dimensional movements in real time using various applications. This technology has been found to be both reliable and valid when measuring range of motion in multiple joints, including cardinal plane range of motion of the cervical spine. ${ }^{9,10}$

There is, however, a gap in the literature demonstrating the efficacy of this technology specifically for measuring UCROM. One inclinometer-based application, Clinometer (plaincode, Stephanskirchen, Germany), uses the device's internal sensors to measure multiplanar movements in real time, with a selfreported measurement accuracy of $\pm 0.1^{\circ}$. This reported accuracy is comparable with the more expensive gold-standard measures used to measure UCROM in a laboratory setting. To our knowledge, no published works collaborate their claims or supporting the use of this particular application to measure UCROM in humans. The purpose of this study was to evaluate the reliability of the Clinometer application in both iPhone (IP) and android (AN) devices when measuring UCROM in a laboratory setting.

\section{Methods}

Two clinically trained doctoral researchers with more than 40 years of combined manual clinical experience performed this laboratory study. 
Each examiner was assigned to measure UCROM using either the IP or AN device and were blinded to their own UCROM measures.

A convenience sample of 38 healthy, college-age students (19 females and 19 males; age: 23.8 [1.2] y) (Table 1) without limited range of motion, stiffness, pain, or injury in the neck or spine for at least 3 months took part in this study. All participants read and signed the appropriate informed consent documentation as required by the Sacred Heart University Institutional Review Board that approved this study.

Order of testing, AN versus IP, was randomized using a coin, with heads indicating AN and tails indicating IP. The direction the subject's head was rotated first was also randomized using a coin, with heads indicating right and tails indicating left.

Subjects were placed in a seated position, as described by Amiri et al, ${ }^{8}$ with their hair suitably positioned to allow visualization of the device and to ensure that the device was properly secured to the head (Universal Head Strap; Velocity Clip, Lake Tahoe, CA) (Figure 1):

1. The smartphone and head strap were positioned on the subject's head, and straps were tightened to prevent movement.

2. The subject's head was passively flexed to end range, with neutral rotation and neutral side bending by the examiner. The head strap position was adjusted until the application read zero,

\section{Table 1 ROM Measurements}

\begin{tabular}{lcc}
\hline & Mean & SD \\
\hline iPhone & & \\
Right rotation & 50.0 & 11.3 \\
Left rotation & 52.1 & 10.8 \\
Total ROM & 102.1 & 21.0 \\
Android & & \\
Right rotation & 47.4 & 6.6 \\
Left rotation & 47.6 & 9.0 \\
Total ROM & 95.0 & 12.7 \\
\hline
\end{tabular}

Abbreviation: ROM, range of motion. Note: Total ROM was calculated by adding mean left and right rotation together.

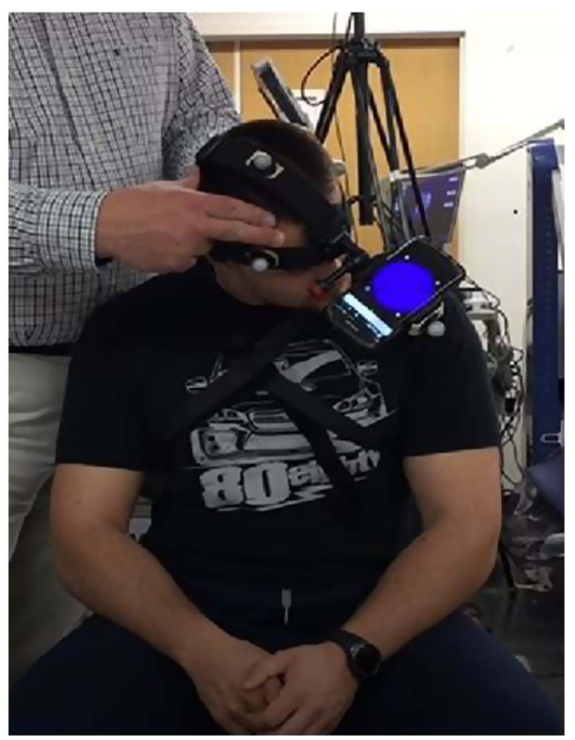

Figure 1 - Testing position. thereby establishing a "neutral" starting position. The subject's head was actively returned to the upright starting position.

3. The subject maximally flexed, extended, rotated right, rotated left, side bent right, and side bent left actively to normalize cervical planes of cardinal motion and ensure that the head strap and phone combination remained securely fixated on the head. The subject's head was actively returned to the upright starting position.

4. The examiner passively flexed the subject's neck to end range.

5. The examiner passively rotated the subject's head in 1 direction until the examiner perceived no further range of motion or the subject reported pain. UCROM was recorded by a research assistant to ensure that the examiner moving the head was unaware of the measures.

6. The subject's head was passively rotated in the other direction and the measure recorded, as above.

7. The subject then returned his or her head to its normal resting position and rested for 20 seconds.

8. Steps 1 to 7 were repeated to obtain 3 trials for that device.

9. The head strap and phone were then removed, and steps 1 through 8 were repeated to obtain 3 trials for the other device.

If at any time the subject or examiner reported or perceived a lack of fixation of the device, the trial was discarded and the entire testing procedure was repeated. This protocol produced 3 measures of UCROM in each direction (left and right) for each device. Data were analyzed using SPSS software (version 24; IBM Corp., Armonk, NY). The intraclass correlation coefficient (ICC) was calculated using a 2-way mixed, absolute agreement model (1) between each examiner-phone and (2) within each examinerphone for the measurements in each rotation direction.

\section{Results}

Total UCROM measures were calculated for each device by adding the average left and average right UCROM measurements. Average of left, right, and total UCROM for each device is provided in Table 1. Data for reliability are presented in Table 2 for interphone/ examiner and intraphone/examiner. A multivariate analysis of variance was performed to determine whether there was a difference in UCROM measurements by sex. Analysis reveals that males had significantly greater left, right, and total UCROM compared with females using either phone $(P<.001)$.

Table 2 Reliability Table

\begin{tabular}{lllll}
\hline & \multicolumn{4}{c}{$\begin{array}{c}\text { Confidence } \\
\text { interval }\end{array}$} \\
\cline { 3 - 4 } & ICC & Upper & Lower & $P$ \\
\hline Interphone/examiner & & & & \\
Mean peak ROM & .872 & .786 & .928 & $<.001$ \\
Mean total ROM & .817 & .575 & .914 & $<.001$ \\
Intraphone/examiner & & & & \\
Android left & .962 & .936 & .979 & $<.001$ \\
Android right & .912 & .849 & .951 & $<.001$ \\
iPhone left & .951 & .917 & .973 & $<.001$ \\
iPhone right & .979 & .964 & .988 & $<.001$ \\
\hline
\end{tabular}

Abbreviations: ICC, intraclass correlation coefficient; ROM, range of motion. 


\section{Discussion}

This published study is the first to measure UCROM using both IP and AN smartphone technology coupled with an inclinometer application. The data demonstrated that UCROM can be reliably measured using these devices in a laboratory setting. The reliability measures for between-phone and within-phone measures were high (ICC > .81) and are consistent with those found in other studies examining the reliability of smartphones coupled with various applications to measure total CROM. ${ }^{9,10}$ This study and previously published reports demonstrate that smartphone technology can reliably measure both total CROM and UCROM. ${ }^{9,10}$ The results of this study suggest that smartphones, using the Clinometer application, can be used to reliably measure UCROM.

This study found that in nonsymptomatic male and female subjects of college age, there was significantly $(P<.001)$ greater left, right, and total UCROM in males compared with females. There appears to be a gap in the literature reporting differences in UCROM differences in males versus females. Ogince et al $^{6}$ noted that males had greater UCROM than females; however, this difference was not statistically significant in a population of subjects with cervicogenic headache. The lack of statistical significance in the Ogince et $\mathrm{al}^{6}$ study may be because their participants were symptomatic and placed in the supine position during measurements, whereas our subjects were sitting, healthy, without complaints of pain or stiffness, and of college age.

The modified flexion and rotation test has been shown to have a $91 \%$ diagnostic accuracy in identifying limitations in UCROM in subjects suffering from cervicogenic headache. ${ }^{6}$ Ogince et $\mathrm{al}^{6}$ relied on expert clinician judgment combined with manual measurement using a modified CROM device to identify those with limited UCROM. This study also relied on expert clinician judgment to determine the end range of cervical rotation while performing the cervical flexion and rotation test in a sitting position and was able to reveal similar data while concurrently measuring UCROM with a smartphone inclinometer application. The cost of the equipment used in this study, excluding the phones themselves, is less than $\$ 15$. This study demonstrated that clinicians can quickly and inexpensively measure UCROM reliably in their patients by using a head strap, smartphone technology, and a commercially available inclinometer application.

\section{Conclusion}

Using a smartphone inclinometer application combined with an inexpensive camera mount can reliably measure UCROM in college-age students.

\section{Acknowledgments}

This work was not funded by an external source. All authors declare no conflicts of interest or financial compensation real or inferred from writing this manuscript.

\section{References}

1. Ernst MJ, Crawford RJ, Schelldorfer S, et al. Extension and flexion in the upper cervical spine in neck pain patients. Man Ther. 2015;20(4): 547-552. PubMed ID: 25578386 doi:10.1016/j.math.2014.12.005

2. Blanpied PR, Gross AR, Elliott JM, et al. Neck pain: revision 2017. J Orthop Sports Phys Ther. 2017;47(7):A1-A83. PubMed ID: 28666405 doi:10.2519/jospt.2017.0302

3. Leddy JJ, Baker JG, Merchant A, et al. Brain or strain? Symptoms alone do not distinguish physiologic concussion from cervical/vestibular injury. Clin J Sport Med. 2015;25(3):237-242. PubMed ID: 25051194 doi:10.1097/JSM.0000000000000128

4. Endo K, Ichimaru K, Komagata M, Yamamoto K. Cervical vertigo and dizziness after whiplash injury. Eur Spine J. 2006;15(6):886890. PubMed ID: 16432749 doi:10.1007/s00586-005-0970-y

5. Reneker JC, Clay Moughiman M, Cook CE. The diagnostic utility of clinical tests for differentiating between cervicogenic and other causes of dizziness after a sports-related concussion: an international Delphi study. J Sci Med Sport. 2015;18(4):366-372. PubMed ID: 24933505 doi:10.1016/j.jsams.2014.05.002

6. Ogince M, Hall T, Robinson K, Blackmore AM. The diagnostic validity of the cervical flexion-rotation test in C1/2-related cervicogenic headache. Man Ther. 2007;12(3):256-262. PubMed ID: 17112768 doi:10.1016/j.math.2006.06.016

7. Hall T, Robinson K. The flexion-rotation test and active cervical mobility-a comparative measurement study in cervicogenic headache. Man Ther. 2004;9(4):197-202. PubMed ID: 15522644 doi:10. 1016/j.math.2004.04.004

8. Amiri M, Jull G, Bullock-Saxton J. Measuring range of active cervical rotation in a position of full head flexion using the $3 \mathrm{D}$ Fastrak measurement system: an intra-tester reliability study. Man Ther. 2003;8(3):176-179. PubMed ID: 12909439 doi:10.1016/ S1356-689X(03)00009-2

9. Tousignant-Laflamme Y, Boutin N, Dion AM, Vallee CA. Reliability and criterion validity of two applications of the iPhone ${ }^{\mathrm{TM}}$ to measure cervical range of motion in healthy participants. J Neuroeng Rehabil. 2013;10(1):69. PubMed ID: 23829201 doi:10.1186/1743-0003-10-69

10. Wellmon RH, Guliek DT, Paterson ML, Guliek CN. Validity and reliability of 2 goniometric mobile apps: device, application, and examiner factors. J Sport Rehabil. 2016;25(4):371-379. PubMed ID: 27632853 doi:10.1123/jsr.2015-0041 
Copyright of Journal of Sport Rehabilitation is the property of Human Kinetics Publishers, Inc. and its content may not be copied or emailed to multiple sites or posted to a listserv without the copyright holder's express written permission. However, users may print, download, or email articles for individual use. 